\title{
Etude d'un jet de ferrofluide confiné en présence de deux sources magnétiques
}

\author{
Walid Nessab ${ }^{1}$, Brahim Fersadou ${ }^{1}$, et Henda Kahalerras ${ }^{1, *}$ \\ ${ }^{1}$ USTHB/FGMGP/LTPMP, BP 32 El Alia 16111 Bab Ezzouar, Alger-Algérie
}

\begin{abstract}
Résumé. La présente étude est une analyse numérique du transfert de chaleur et de l'écoulement d'un jet de ferrofluide confiné dans un canal plan en présence de deux sources magnétiques. Le modèle de Buongiorno est utilisé pour décrire l'écoulement du ferrofluide avec la prise en compte de l'effet ferrohydrodynamique. Les équations gouvernantes avec les conditions aux limites associées sont résolues par la méthode des volumes finis. Les résultats révèlent une amélioration du transfert de chaleur avec l'augmentation du nombre magnétique $(\mathrm{Mn})$ et la réduction du rapport d'ouverture (R).
\end{abstract}

\section{Introduction}

Différentes techniques ont été développées pour améliorer le transfert de chaleur dans les systèmes industriels. Parmi ces méthodes, nous pouvons citer l'utilisation des fluides magnétiques qui sont des mélanges de fluides de base classiques et de nanoparticules de Fe, $\mathrm{Co}$ ou $\mathrm{Fe}_{3} \mathrm{O}_{4}$. Leurs propriétés attractives ont fait que ces fluides peuvent être appliqués dans de nombreux domaines tels que le génie thermique, l'emballage électronique, la bioingénierie, etc. Plusieurs travaux de recherche ont été menés ces dernières années [1-6] et ont mis en évidence les améliorations apportées à divers systèmes thermiques par utilisation des ferrofluides qui représentent une classe des fluides magnétique. C'est dans ce contexte que s'inscrit ce travail qui consiste à examiner numériquement le problème de la convection forcée d'un jet de ferrofluide (eau- $\mathrm{Fe}_{3} \mathrm{O}_{4}$ ) confiné dans un canal plan en présence de deux sources magnétiques.

\section{Modèle physique et formulation mathématique}

Le domaine physique étudié est représenté sur la Fig. 1.

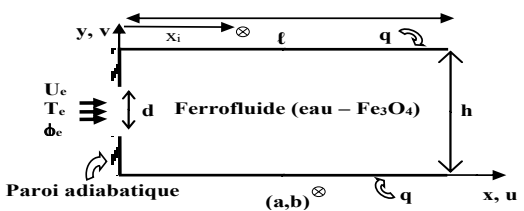

Fig. 1. Domaine physique

*Auteur correspondant : hkahalerras@usthb.dz 
En adoptant le modèle de Buongiorno [7] avec prise en compte de l'effet ferrohydrodynamique, les équations de conservation s'écrivent de la manière suivante :

$$
\begin{gathered}
\frac{\partial U}{\partial X}+\frac{\partial V}{\partial Y}=0 \\
U \frac{\partial U}{\partial X}+V \frac{\partial U}{\partial Y}=-\frac{\partial P}{\partial X}+\frac{R}{\operatorname{Re}} \frac{\rho_{f}}{\rho_{n f}}\left[\frac{\partial}{\partial X}\left(\frac{\mu_{n f}}{\mu} \frac{\partial U}{\partial X}\right)+\frac{\partial}{\partial Y}\left(\frac{\mu_{n f}}{\mu} \frac{\partial U}{\partial Y}\right)\right]+\frac{\rho_{f}}{\rho_{n f}} M n\left(\omega_{c}-\theta-\omega_{e}\right) \bar{H} \frac{\partial \bar{H}}{\partial X} \\
U \frac{\partial V}{\partial X}+V \frac{\partial V}{\partial Y}=-\frac{\partial P}{\partial Y}+\frac{R}{\operatorname{Re}} \frac{\rho_{f}}{\rho_{n f}}\left[\frac{\partial}{\partial X}\left(\frac{\mu}{\mu} \frac{\partial V}{\partial X}\right)+\frac{\partial}{\partial Y}\left(\frac{\mu_{n f}}{\mu} \frac{\partial V}{\partial Y}\right)\right]+\frac{\rho_{f}}{\rho_{n f}} M n\left(\omega_{c}-\theta-\omega_{e}\right) \bar{H} \frac{\partial \bar{H}}{\partial Y} \\
\begin{aligned}
U \frac{\partial \theta}{\partial X}+V \frac{\partial \theta}{\partial Y}=\frac{R}{\operatorname{RePr}} \frac{\left(\rho C_{p}\right)_{f}}{\left(\rho C_{p}\right)_{n f}}\left[\frac{\partial}{\partial X}\left(\frac{k_{n f}}{k_{f}} \frac{\partial \theta}{\partial X}\right)+\frac{\partial}{\partial Y}\left(\frac{k_{n f}}{k_{f}} \frac{\partial \theta}{\partial Y}\right)+N_{B}\left(\frac{\partial \varphi}{\partial X} \frac{\partial \theta}{\partial X}+\frac{\partial \varphi}{\partial Y} \frac{\partial \theta}{\partial Y}\right)+N_{T}\left(\left(\frac{\partial \theta}{\partial X}\right)^{2}+\left(\frac{\partial \theta}{\partial Y}\right)^{2}\right)\right] \\
+\frac{\left(\rho C_{p}\right)_{f}}{\left(\rho C_{p}\right)_{n f}} M n E c\left(+\omega_{e}\right) \bar{H}\left[U \frac{\partial \bar{H}}{\partial X}+V \frac{\partial \bar{H}}{\partial Y}\right]
\end{aligned} \\
U \frac{\partial \varphi}{\partial X}+V \frac{\partial \varphi}{\partial Y}=\frac{R}{\operatorname{Re} \operatorname{Pr}}\left[\frac{\partial}{\partial X}\left(\frac{1}{L e} \frac{\partial \varphi}{\partial X}\right)+\frac{\partial}{\partial Y}\left(\frac{1}{L e} \frac{\partial \varphi}{\partial Y}\right)+\frac{\partial}{\partial X}\left(\frac{1}{L e} \frac{N_{T}}{N_{B}} \frac{\partial \theta}{\partial X}\right)+\frac{\partial}{\partial Y}\left(\frac{1}{L e} \frac{N_{T}}{N_{B}} \frac{\partial \theta}{\partial Y}\right)\right]
\end{gathered}
$$

\section{Modélisation numérique}

Les équations gouvernantes sont résolues numériquement par la méthode des volumes finis [8]. Le schéma loi de puissance est adopté dans la procédure de discrétisation afin de traiter les termes convectifs et diffusifs. Les équations algébriques sont résolues par la technique ligne par ligne et un maillage non-uniforme est employé avec un système de grille de $300 \times$ 70 (dans les directions $\mathrm{X}$ et $\mathrm{Y}$, respectivement). Comme critère de convergence du processus itératif, l'erreur relative sur chaque variable entre deux itérations successives doit être inférieure à $10^{-6}$.

\section{Résultats}

La Fig. 2 illustre que sous l'effet FHD, deux zones de recirculation prennent naissance au niveau des sources avec accroissement de leurs tailles quand Mn augmente. Ces zones tourbillonnaires perturbent le développement des couches limites thermiques; ce qui aura un effet favorable sur le transfert de chaleur. En effet, la Fig. 3 montre une amélioration des échanges thermiques entre le ferrofluide et les deux parois du canal en augmentant la valeur du nombre magnétique. Quant à l'effet de $X_{\mathrm{i}}$, il apparait qu'il est préférable de placer les deux sources en quinconce et en rapprochant la source supérieure de l'entrée du canal.
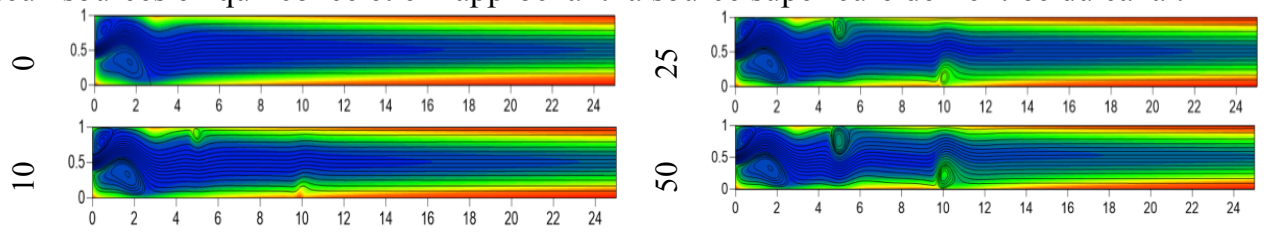

Fig. 2. Lignes de courant et isothermes pour différents $M n$ à $R=1 / 4$ et $X_{i}=5$
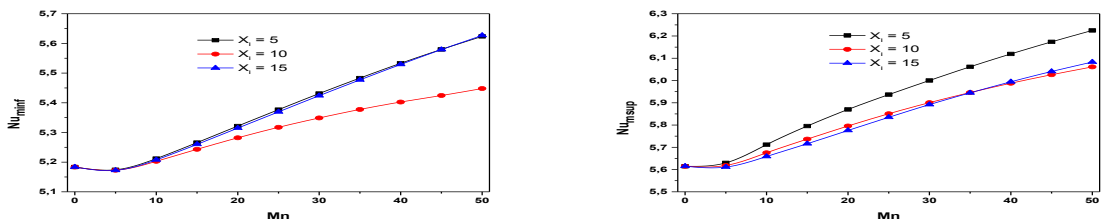

Fig. 3. Variation des nombres de Nusselt moyens avec Mn à $\mathrm{R}=1 / 4$ 
L'effet du rapport d'ouverture est représenté sur les Fig. 4 et Fig. 5. Pour $R \leq 1 / 2$, la structure d'écoulement est symétrique avec présence de deux tourbillons contrarotatifs juste à l'entrée du canal. En déca de cette valeur de R, il y'a augmentation de la taille de ces deux vortex mais de manière asymétrique et le jet est alors dévié vers la paroi supérieure ensuite vers la paroi inferieure. La réduction du rapport d'ouverture a un effet similaire, sur la structure de l'écoulement au niveau des sources, que l'augmentation de $\mathrm{Mn}$; c'est-à-dire l'accroissement de la taille des zones tourbillonnaires. Ceci agit alors positivement sur les valeurs des nombres de Nusselt moyens qui augmentent avec la diminution de R comme l'illustre la Fig. 5. L'influence de la position de la source supérieure ne devient perceptible que pour $\mathrm{R} \leq 1 / 4$.

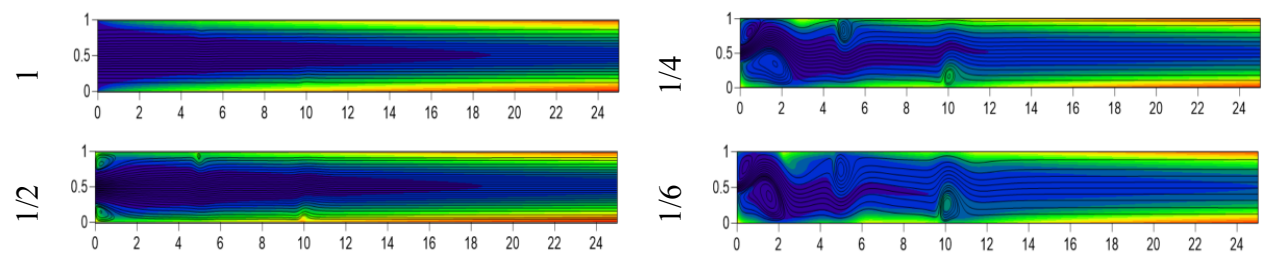

Fig. 4. Lignes de courant et isothermes pour différents $\mathrm{R}$ à $\mathrm{Mn}=30$ et $\mathrm{X}_{\mathrm{i}}=5$
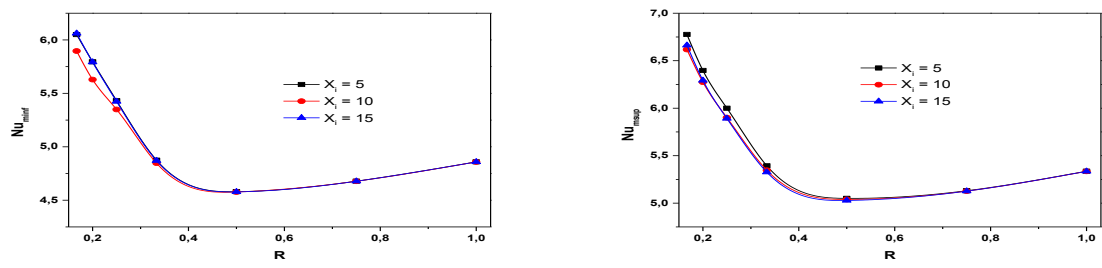

Fig. 5. Variation des nombres de Nusselt moyens avec $\mathrm{R}$ à $\mathrm{Mn}=30$

\section{Conclusion}

Le présent travail est une simulation numérique de la convection forcée d'un jet de ferrofluide confiné dans un canal plan sous l'influence de deux sources magnétiques. Les résultats ont été analysés en mettant en évidence l'influence de l'intensité des sources magnétiques traduite par $\mathrm{Mn}$, la hauteur du jet à l'entrée du canal donnée par $\mathrm{R}$, et la position de la source supérieure $\mathrm{X}_{\mathrm{i}}$. Les zones tourbillonnaires créées au niveau des sources et du rétrécissement à l'entrée du canal ont conduit à une amélioration des taux de transfert de chaleur. Les nombres de Nusselt moyens augmentent avec l'accroissement de $\mathrm{Mn}$ et la réduction de $\mathrm{R}$. La meilleure disposition des sources est celle où elles sont en quinconce avec la source supérieure rapprochée de l'entrée du canal.

\section{Références}

1. G.A. Maugin, Int. J. Eng. Sci. 31 (1993)

2. R. Ganguly, S. Sen, I.K. Puri, J. Magnetism Magnetic Mater. 271 (2004)

3. Y. Xuan, Q. Li, M. Ye, Int. J. Therm. Sci. 46 (2007)

4. A. Ghofrani, M.H. Dibaei, A.H. Sima, M.B. Shafii, Exp. Therm. Fluid Sci. 49 (2013)

5. M. Sheikholeslami, T. Hayat, A. Alsaedi, Int. J. Heat Mass Transfer 106 (2017)

6. M. Sheikholeslami, S.A. Shehzad, Int. J. Heat Mass Transfer 118 (2018)

7. J. Buongiorno, ASME J. Heat Transfer 128 (2006)

8. S.V. Patankar. McGraw Hill (1980) 